Article

\title{
Functional Analysis of an Essential GSP1/Ran Ortholog Gene, CpRan1, from the Chestnut Blight Fungus Cryphonectria parasitica Using a Heterokaryon
}

\author{
Yo-Han Ko, Jeesun Chun and Dae-Hyuk Kim *
}

check for updates

Citation: Ko, Y.-H.; Chun, J.; Kim, D.-H. Functional Analysis of an Essential GSP1/Ran Ortholog Gene, CpRan1, from the Chestnut Blight Fungus Cryphonectria parasitica Using a Heterokaryon. J. Fungi 2021, 7, 332. https://doi.org/10.3390/jof7050332

Academic Editor: Paola Bonfante

Received: 12 March 2021

Accepted: 22 April 2021

Published: 25 April 2021

Publisher's Note: MDPI stays neutral with regard to jurisdictional claims in published maps and institutional affiliations.

Copyright: (c) 2021 by the authors. Licensee MDPI, Basel, Switzerland. This article is an open access article distributed under the terms and conditions of the Creative Commons Attribution (CC BY) license (https:/ / creativecommons.org/licenses/by/ $4.0 /)$.
Department of Molecular Biology, Institute for Molecular Biology and Genetics, Jeonbuk National University, 567 Baekje-daero, Jeonju, Chonbuk 54896, Korea; kocineda@naver.com (Y.-H.K.); brainyjsc@gmail.com (J.C.)

* Correspondence: dhkim@jbnu.ac.kr; Tel.: +82-63-270-3440; Fax: +82-63-270-4312

\begin{abstract}
Functional analysis of a GSP1/Ran ortholog, CpRan1, from Cryphonectria parasitica was conducted. Genotype analysis revealed that the putative CpRan1-null mutant was a heterokaryotic transformant harboring two different types of nuclei, one with the wild-type CpRan1 allele and the other with the CpRan1-null mutant allele. The mycelial growth and colony morphology of the heterokaryotic transformant was normal. Microscopic analysis of the resulting conidia (aseptate and monokaryotic asexual spores) demonstrated that although normal germinating spores were observed from conidia harboring a nucleus with the wild-type $C p R a n 1$ allele, a number of residual conidia that did not germinate existed. Complementation analysis using protoplasts from the heterokaryon with the wild-type CpRan1 allele confirmed that the CpRan1 gene is essential to C. parasitica. Complementation analysis using the various $C p R a n 1$ chimera constructs allowed us to perform a functional analysis of essential amino acids of the CpRan1. Among the four suggested essential amino acids, Lys-97 for ubiquitination was determined to not be an essential residue. Moreover, the CpRan1-null mutant allele was successfully complemented with mouse Ran gene, which suggested that the biological function of Ran gene is evolutionary conserved and that our heterokaryon rescue can be applied for the functional analysis of heterologous genes.
\end{abstract}

Keywords: Cryphonectria parasitica; Ran; heterokaryon; hypovirulence

\section{Introduction}

Cryphonectria parasitica (Murrill) Barr, causes chestnut blight and has devastated chestnut forests and orchards in North America since the early 20th century [1]. However, C. parasitica, which contains a cytoplasmic single stranded RNA (ssRNA) virus, Cryphonectria hypovirus 1 (CHV1), has had lowered virulence, a phenomenon referred to as "hypovirulence" [2-4]. CHV1-infected C. parasitica displays diverse hypovirulence-associated symptoms, such as reduced pigmentation, sporulation, laccase production, and oxalate accumulation [5-7]. More interestingly, CHV1 can be transferred from the virus-containing hypovirulent strain to virus-free strain during hyphal fusion resulting in hypovirulence and its associated symptoms, which is an effective good model for naturally occurring biological control by mycovirus, also known as virocontrol $[8,9]$.

Molecular analysis of C. parasitica-hypovirus interactions allowed us to ascribe the development of these viral symptoms to aberrant expressions of specific genes in the hypovirulent strain [10-15]. Due to the efficient genetic manipulations of C. parasitica, the availability of a high-quality C. parasitica genome sequence (http: / / genome.jgi-psf.org/ Crypa2/Crypa2.home.html, accessed on 16 November 2016), and the application of an infectious cDNA copy of the hypovirus, C. parasitica-hypovirus interactions have been considered to be an ideal model to investigate fungus-virus interactions [16-20].

The Ran (Ras-related nuclear) protein is a highly conserved small GTP-binding nuclear protein and a vital component of the nucleocytoplasmic transport machinery that moves 
into and out of the cell nucleus during interphase and mitosis. In turn, Ran is known to play an important role in numerous cellular processes including various mitotic processes as well as antiviral immunity [21-25]. Ran primarily localizes in the nucleus and only a minor proportion is cytosolic in interphase cells, translocating between the cytosol and the nucleus through nuclear pore complexes. The concentration of RanGTP between the nucleus and cytosol is important for the directionality of nucleocytoplasmic trafficking. Nucleotide binding to Ran is tightly regulated by modulators such as Ran-binding proteins (RanBPs), guanine nucleotide exchange factor (RanGEF), and RanGTPase-activating protein (RanGAP). The strict compartmentalization of these modulating proteins allows them to maintain a sharp gradient in the concentration of RanGTP between the nucleus and the cytosol [26,27]. Recently, we have described how the gene encoding Ran-binding protein of $C$. parasitica, CpRbp1, was affected by CHV1 infection or supplementation with tannic acid, which is known to be abundant in the bark of chestnut trees and is defined as being one of the major barriers against pathogen infection [20]. Our functional analysis proved that $C p R b p 1$ is essential to biological processes [28].

Heterokaryosis, defined as the presence of two or even more genetically different nuclei in a common cytoplasm, is a unique genetic feature of fungi. A heterokaryon refers to a fungus that exhibits heterokaryosis by maintaining different types of nuclei in a cell. Heterokaryon is a useful genetic resource for nonsexual genetic variation. Although the ratio of nuclear genotypes in heterokaryons varies with a wide range and can be influenced by environmental conditions, a heterokaryon is effective in maintaining and even proliferating nuclei that contain a lethal genotype. In addition, heterokaryons can break down during the production of uninucleate spores, each of which can be cultured as a monokaryotic progeny. The maintaining, proliferating, and breaking down of different genotypes is specifically appropriate for the analysis of mutant nuclei in which a gene of interest is severely detrimental or lethal. These genetic processes provide the means of functional analysis via complementation with various chimeric constructs of the corresponding gene [28].

Regarding heterokaryon analysis, C. parasitica has the following advantages over other fungi: first, analysis of the natural population of $C$. parasitica reveals the presence of stable heterokaryons [29]; second, forced heterokaryon formation with mutant nuclei of an essential gene is successful during genetic manipulation [28]; third, the asexual spore of C. parasitica is a uninucleate single cell $[30,31]$. C. parasitica has a relatively high tendency to form heterokaryons, stably propagate them, and then easily break down each nuclei. These characteristics make this fungal system ideal for performing the functional analysis of morphogenic as well as essential genes.

Our previous pathoproteomic studies demonstrated that not all, but specific components of the Ran complex, such as RanBP1 and Ran, were affected by CHV1 infection and/or tannic acid supplementation. Although our recent study on RanBP revealed that the CpRbp1 encoding RanBP is essential, no studies on the other components of the Ran complex have been conducted. Thus, we investigated the biological function of the Ran gene, which is also affected by CHV1 infection and tannic acid supplementation [20]. In this study, we describe heterokaryon formation with Ran gene mutant nuclei. We also detail our functional analysis of the Ran gene via complementation of the mutant nucleus with various Ran gene structures.

\section{Materials and Methods}

\subsection{Fungal Strains and Growth}

Strains of virus-free C. parasitica EP155/2 (ATCC 38755) and its genetically identical (isogenic) CHV1-713-containing hypovirulent UEP1 were maintained on a potato dextrose agar supplemented with L-methionine $(0.1 \mathrm{mg} / \mathrm{mL})$ and biotin $(0.1 \mu \mathrm{g} / \mathrm{mL})(\mathrm{PDAmb})$ at $25^{\circ} \mathrm{C}$ with constant low light [18]. Endothia Parasitica (EP) complete medium was used for standard liquid culture [32]. The methods to prepare the primary inoculum for liquid cultures and culture conditions have been previously described [18]. Tannic acid induction 
of fungal strains was performed according to procedures previously described [20]. Briefly, mycelia cultured for 10 days on cellophane that had been layered on the top of PDAmb medium were transferred to a plate supplemented with an appropriate concentration of tannic acid [33]. Consistent with previous procedures, the mycelium was collected and lyophilized until it was used [34].

\subsection{Quantitative Analysis of Transcript Accumulation Using Real-Time RT-PCR}

To examine $C p R a n 1$ gene expression levels, quantitative real-time PCR using reverse transcriptase (qRT-PCR) was performed using a GeneAmp 7500 sequence detection system (Applied Biosystems, Foster City, CA, USA) and a SYBR green mixture RT kit (Applied Biosystems, Foster City, CA, USA) as previously described [28]. Glyceraldehyde3-phosphate dehydrogenase gene (Gpd) was used as an internal control $[4,10,28,30,31]$. Primer pairs for Gpd and CpRan1 genes were indicated as RT-Gpd-F (forward) and RTGpd-R (reverse), as well as RT-Ran1-F1 (forward) and RT-Ran1-R1 (reverse), respectively (Supplementary Table S1). In the sample, transcript abundance, relative to the amount of $\mathrm{Gpd}$ gene, was calculated using the comparative threshold cycle method as previously described [35]. Analyses were conducted from three independent RNA preparations, in triplicate for each transcript, with primers specific for the Gpd and CpRan1 genes.

\subsection{Statistical Analysis}

The accumulation of $C p R a n 1$ transcripts of each strain were compared with hypovirus infection or tannic acid supplementation, analyzed by ANOVA using SPSS software (version 23.0, SPSS Inc., Chicago, IL, USA), and then comparison significance was determined using the Tukey honest significant difference (HSD) test at $p<0.01$.

\subsection{Cloning and Characterization of a GSP1/Ran Like Gene, CpRan1}

Tandem mass analysis revealed the amino acid sequence of a selected protein spot [20]. The genome data base of C. parasitica (http:/ / genome.jgi-psf.org/Crypa2/Crypa2.home. $\mathrm{html}$, accessed on 16 November 2016) was analyzed to identify the gene encoding the determined amino acid sequence. In this study, we further investigated one of these spots corresponding to the Saccharomyces cerevisiae GSP1. We performed PCR amplification of this gene using primers CpRan1-gF1 (forward) and CpRan1-gR1 (reverse) (Supplementary Table S1). The resulting 5000-bp PCR amplicon was cloned into the pGEM-T Easy vector and sequenced.

To obtain the cDNA clone, PCR using reverse transcriptase (RT-PCR) was performed with primers CpRan1-cF1 (forward) and CpRan1-cR1 (reverse) (Supplementary Table S1). The resulting 651-bp cDNA amplicon was cloned and sequenced.

\subsection{Southern and Northern Blot Analysis}

Genomic DNA extraction and Southern blot analysis, implemented with the restriction enzyme EcoRV and a radioactive probe, were conducted as described in an earlier study [16].

RNA extraction from liquid cultures was conducted as previously described [36]. RNA extraction from mycelia mats grown on cellophane layered on top of appropriate solid media was performed as previously described [20]. The level of CpRan1 transcript was normalized to that of glyceraldehyde-3-phosphate dehydrogenase $(\mathrm{Gpd})$ of $\mathrm{C}$. parasitica as an internal control for gene expression [37].

\subsection{Target Gene Replacement Vectors and Fungal Transformation}

Gene replacement using split-marker deletion cassettes was applied to study the biological function of the CpRan1 gene [38]. Two molecular DNA cassettes, each of which contained a part of the hygromycin B phosphotransferase gene cassette $(h p h)$ fused the either the 2643-bp 5'- or 2576-bp 3'- flanking regions of the CpRan1 gene, were prepared by overlap PCR [38] as follows: a 1055-bp PCR amplicon containing the 1035-bp 5' flanking 
region of CpRan1 was amplified using gene-specific primers Ran 5'-F1 and Ran 5'-R1 (Supplementary Table S1). A 1627-bp part of a selection marker gene containing the promoter and part of the N-terminus was amplified using primers Ran-Hph-F1 and HphR1 (Supplementary Table S1). The fusion of these two PCR amplicons was conducted using overlap extension PCR with primers Ran1-F1 and Hph-R1. A 2576-bp PCR fragment of the 980-bp $3^{\prime}$ flanking region and a 1596-bp part of the selection marker gene containing the terminator and part of the C-terminus were amplified and fused using primers HphF2/Hph-Ran-R2, Ran 3'-F2/Ran 3'-R2, and Hph-F2/Ran 3'-R2 (Supplementary Table S1). The resulting molecular cassettes were then used simultaneously to transform protoplasts of the C. parasitica $\mathrm{EP} 155 / 2$ strain.

Functional complementation of the CpRan1-null mutant using the wild-type allele was performed. The complementing vector $\mathrm{pCRan}$ was constructed by insertion of a 4615-bp NotI fragment of pSilent-Dual1G (pSD1G), which contained the geneticin resistance cassette [39], into NotI-digested pRan carrying a 5035-bp fragment with the full-length $C p R a n 1$ gene. The resulting vector was then used to transform the putative CpRan1-null mutant.

Protoplast preparation and transformation were performed as previously described $[16,18]$. Transformants were selected from agar plates that were supplemented with $150 \mu \mathrm{g} / \mathrm{mL}$ hygromycin B (Calbiochem, San Diego, CA, USA) or $150 \mu \mathrm{g} / \mathrm{mL}$ geneticin (Invitrogen, Carlsbad, CA, USA), subcultured three to four times on appropriate selective media, and single spores were isolated when possible, as previously described [28,30]. PCR and Southern blot analysis of the transformants were conducted to confirm replacement and in-trans complementation of the CpRan1 gene.

\subsection{Staining and Microscopy}

DAPI staining was performed as described previously [31,40]. Before imaging, adherent mycelia were fixed on coverslips with formaldehyde and washed briefly with distilled water. Mycelial staining was performed for $10 \mathrm{~min}$ at room temperature using $100 \mathrm{ng} / \mathrm{mL}$ 4,6-diamidino-2-phenylindole (DAPI, Sigma-Aldrich, St. Louis, MO, USA) and was followed by washing with distilled water. The slides were then mounted in phosphate buffered saline (PBS) containing 50\% glycerol and $0.1 \%$ n-propyl-gallate and observed under a fluorescence microscope using a model LSM 880 in the Center for University-wide Research Facilities (CURF) at Jeonbuk National University (Carl Zeiss, Germany).

\subsection{Ratio of Different Types of Nuclei}

To estimate the ratio of different types of nuclei in heterokaryons, the putative heterokaryotic transformant was transferred every 5 days over 10 times on non-selective PDAmb and hygromycin B-containing selective PDAmb media. Each mycelium was grown on cellophane overlaid on the top of the appropriate media and harvested to extract genomic DNA. The nuclear ratio was confirmed through Southern blot analysis. The density of each band was quantified using Image J software (provided by the National Institutes of Health). In order to obtain conidia, successively cultured strains were transferred onto PDAmb and incubated for 2 weeks. Then, 100 conidia (as determined by a hemocytometer) were spread on PDAmb plates supplemented with or without hygromycin $B$. The resulting colony-forming units (CFUs) were counted to determine the number of wild-type nuclei. Three representative plates were selected for each transfer and analyzed statistically.

\subsection{Construction of Complementary Vectors}

Complementation assays of the CpRan1-null mutant were performed using genomic DNA, cDNA of CpRan1, and Ran cDNA of the mouse Mus musculus. The complementing vector based on genomic DNA, pCRan-gDNA, was constructed by insertion of a 5615-bp NotI fragment of pBluescript SK(-) containing the geneticin resistance cassette [39] into NotI-digested pGRan carrying a 5035-bp fragment with the full-length CpRan1 gene. The 
complementing vectors based on cloned cDNA of $C p R a n 1$ were constructed by insertion of a 1425-bp NotI-digested cDNA expression cassette consisting of a strong constitutive cryparin promoter (p188), 651-bp cDNA, along with a trpC terminator into a 4615-bp NotI fragment of the geneticin resistance cassette. In order to analyze the role of the selected amino acid residues in the protein product of the CpRan1 gene, we used the Saccharomyces Genome Database (SGD; https: / / www.yeastgenome.org/locus/S000004284/protein, accessed on 14 May 2018) to select amino acid residues with post-translational modifications. Among seven suggested residues, we randomly selected four involved in succinylation (K25), ubiquitination (K101), ubiquitination (K125), and phosphorylation (S155) for analysis. In silico analysis using pair-wise comparison of amino acid sequences from GSP1 and CpRan1 genes revealed corresponding conserved amino acid residues for succinylation (at K21), ubiquitination (at K97), ubiquitination (at K121), and phosphorylation (at S151) within the protein product of the $C p R a n 1$ gene. Four different chimeric structures harboring a point mutation at an appropriate amino acid residue were obtained by PCR amplification using a primer set that contained the corresponding mutated sequence. The complementing vector for the cDNA of mouse Ran was also constructed as that of the cDNA of $C$. parasitica. The resulting vectors were then used to transform the putative CpRan1-null mutant.

\section{Results}

\subsection{Characterization of a Tannic Acid-Responsive and Hypoviral-Regulated CpRan1 Gene}

Proteomic analysis of $C$. parasitica revealed a protein spot, which increased due to the hypovirus infection or tannic acid supplementation, and was identified as a Ran protein (GSP1/Ran) ortholog [20]. Inspection of the C. parasitica genome database (http://genome. jgi-psf.org/Crypa2/Crypa2.home.html, accessed on 16 November 2016) allowed us to determine the corresponding nucleotide sequence that encode the Ran protein ortholog. PCR amplification of a $5000 \mathrm{bp}$ fragment, which was expected to contain the full-length Ran gene, followed by cloning and sequencing confirmed the nucleotide sequence. A near full-length cDNA was amplified using reverse transcription polymerase chain reaction (RT-PCR) with the primer pair CpRan1-cF1 and CpRan1-cR1 at 1 to 18 and 1031 to 1048. The resulting $656 \mathrm{bp}$ amplicon was cloned and sequenced. Sequence comparison of the cDNA clone with the corresponding genomic clone revealed that the cDNA clone consisted of five exons, with four intervening sequences of 64 to 241,335 to 396, 551 to 622, and 829 to 908 (relative to the start codon). These were equal to the computer prediction. The sequence around the first ATG was in good agreement with Kozak's consensus sequence in that the nt -3 position was the A in CAACGATCATG. The putative poly (A) signal of AAATA was observed at $365 \mathrm{nt}$ downstream of the stop codon.

The cloned gene has an open reading frame (ORF) of $651 \mathrm{bp}$ that encodes 217 amino acid residues with an estimated molecular mass of $24.5 \mathrm{kDa}$ and a pI of 6.11 (GenBank No. KAF3769082.1). Homology searches using the deduced amino acid sequence indicated that the protein product of the cloned gene is related to other fungal Ran homologs from Trichoderma virens (XP_013951777.1, 94.91\% AA identity), Fusarium beomiforme (KAF4331629.1, 94.44\%), Colletotrichum asianum (KAF0317623.1, 93.95\%), Verruconis gallopava (XP_016218807.1, 92.89\%), and Aspergillus nidulans (AAR08135.1, 91.04\%). The multiple alignment of five closely related Ran proteins showing an $\mathrm{E}$ value $\approx 0.0$ and Ran genes from S. cerevisiae revealed that the protein product of the cloned gene had a characteristic multi-domain structure, which consisted of four guanine nucleotide-binding domains, an effector domain, and an acidic C-terminal domain (Supplementary Figure S1A). The phylogram was supported with high bootstrap values suggesting a genuine evolutionary relationship (Supplementary Figure S1B). Together with the presence of conserved domains and a significant homology to known fungal Rans, the cloned gene was referred to as $C p R a n 1$ for the C. parasitica Ran gene. In addition, inspection of the C. parasitica genome database revealed that no other gene contained the characteristic multi-domains and no gene except $C p R a n 1$ showed high similarity to other known Ran proteins, which strongly indicated that the CpRan1 gene is the only gene encoding the Ran protein. 


\subsection{Expression of $C p R a n 1$}

Since our previous proteomic study showed down-regulation of the protein product of the CpRan1 gene by either hypovirus infection or tannic acid supplementation [20], the accumulation of the CpRan1 transcript of the wild-type EP155/2 strain and its isogenic CHV1-infected hypovirulent strain UEP1 were examined under corresponding conditions using qRT-PCR and Northern blot analysis (Figure 1A and Supplementary Figure S2). The qRT-PCR results indicated that a slight but significant increase in CpRan1 transcript levels occurred in EP155/2 up to $24 \mathrm{~h}$ after the TA-supplementation. EP155/2 induction levels greatly increased at $36 \mathrm{~h}$ and $48 \mathrm{~h}$ after TA-supplementation. The accumulation of $C p R a n 1$ transcripts increased even more significantly in the CHV1-infected UEP1 strain, which is isogenic to the wild type. No further induction, however, was observed in CHV1-infected UEP1 cultured on TA-supplemented media. Northern blot analysis revealed that the accumulation of CpRan 1 transcripts of the wild-type EP155/2 was increased $24 \mathrm{~h}$ after the TA supplementation. CpRan1 transcript increases were also noted in UEP1, a slight rise being observed in UEP1 with the TA supplementation (Supplementary Figure S2). These results are consistent with those obtained through the qRT-PCR. Interestingly, a significant reduction in the accumulation of CpRan1 transcripts was observed after $36 \mathrm{~h}$ in CHV1-infected UEP1 cultured on TA-supplemented media. These results indicate that hypovirus infection or TA supplementation alone upregulated the accumulation of the $C p R a n 1$ transcript in $C$. parasitica. Their effect, however, on $C p R a n 1$ expression was counteracted in combination. These results suggest that the expression of the CpRan1 gene in planta is greatly affected by the CHV1 infection compared to its expression with the virus-free EP155/2. This interaction suggests that the expression of the CpRan1 gene is important for fungal virulence.
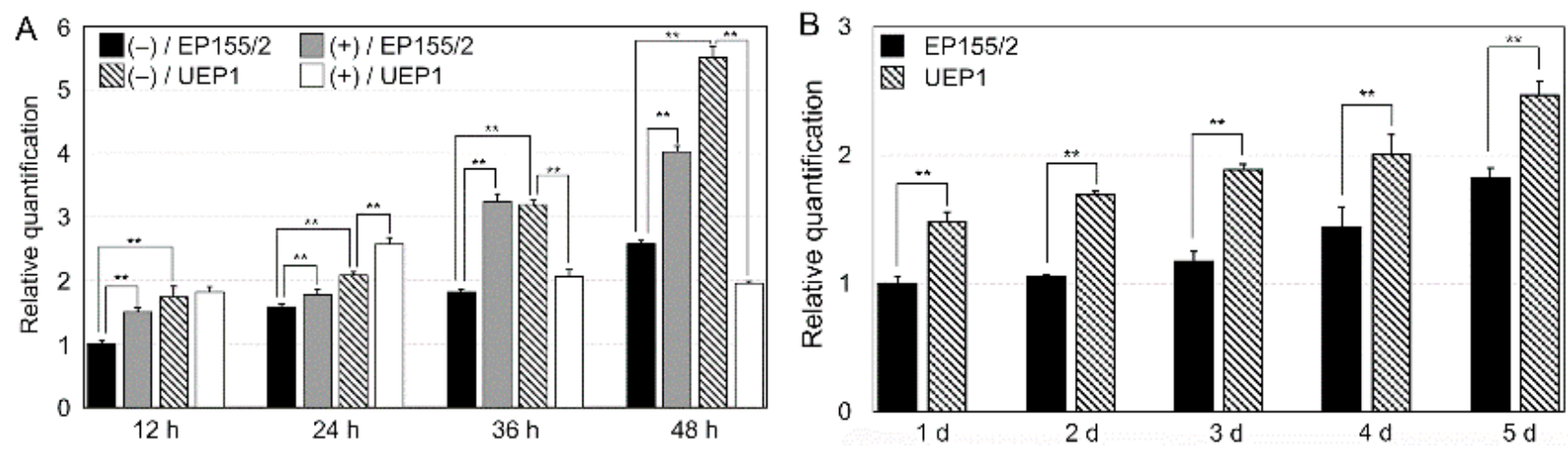

Figure 1. Expression analysis of CpRan1. (A) qRT-PCR analysis of the expression levels of CpRan1 in response to CHV1 infection and TA supplementation. Changes in expression of CpRan1 between the wild-type (EP155/2; indicated as solid bars) and CHV1-infected hypovirulent (UEP1; indicated as slashed bars) strains relative to levels of glyceraldehyde-3phosphate dehydrogenase $(g p d)$ are indicated; $(+)$ and $(-)$ indicate with and without TA supplementation, respectively. (B) qRT-PCR analysis of the expression levels of $C p R a n 1$ during cultivation in standard liquid EP complete medium. Numbers at the bottom indicate hours after the transfer $(\mathbf{A})$ and days in liquid culture $(\mathbf{B}) .{ }^{* *}$ indicates a significant change at $p<0.01$. Error bars indicate standard deviation based on three independent measurements.

We also examined the accumulation of transcripts of the CpRan1 gene of the wildtype EP155/2 and CHV1-infected UEP1 strains under standard liquid-culture conditions (Figure 1B). The accumulation of the CpRan1 transcript gradually increased in both EP155/2 and UEP1 strains as the culture proceeded. Significant upregulation of the CpRan1 transcript was observed in the CHV1-infected UEP1 strain.

\subsection{Screening and Identification of a CpRan1-Null Mutant}

Understanding the biological function of the $C p R a n 1$ gene was initiated by a comparison of the wild type with the CpRan1-null mutant, which was constructed by site-directed recombination during integrative transformation. A total of 142 putative transformants were obtained from agar plates that were overlaid with $150 \mu \mathrm{g} / \mathrm{mL}$ hygromycin B and 
passaged four times on selective media containing $50 \mu \mathrm{g} / \mathrm{mL}$ hygromycin B by successively transferring agar plugs containing actively growing young hyphae. All actively growing stable transformants were selected for further analysis. A total of 130 stable transformants were further screened by PCR using two pairs of primer sets designed for the detection of the mutant allele of the CpRan1 gene, i.e., outer gene-specific and inner $h p h$ primers (Primers 1 and 4 and 3 and 2 in Figure 2A) corresponding to -1988 to $-1967,1222$ to 1241,446 to 465 , and 2991 to 3012 (relative to the start codon of the CpRan1 and hph genes). With one exception, all transformants showed the absence of PCR amplicons. The exception showed PCR amplicons of the expected sizes of $3608 \mathrm{bp}$ and $3561 \mathrm{bp}$ corresponding to the disrupted alleles of the CpRan1 gene (Supplementary Figure S3A,B). When the putative CpRan1-null mutant was further examined by Southern blot analysis, however, two hybridizing bands were observed. One of these bands was the same size as the wild-type CpRan1 allele, while the other band was the size of the expected CpRan1-null allele (Figure 2B). In addition, PCR analysis designed for the amplification of the replaced fragment by using outer gene-specific primers (Primers 1 and 2 in Figure 2A) revealed not one, but two bands presumably representing the wild-type allele and the mutant allele (Supplementary Figure S3C). Subsequent cloning and sequencing of these two PCR amplicons confirmed that the $5000 \mathrm{bp}$ amplicon was the wild-type allele and the 6373 bp amplicon was the CpRan1-null mutant allele that replaced the CpRan1 gene. The CpRan1-null mutant allele was substituted with the replacement vector lacking the genomic region between $13 \mathrm{bp}$ and $1047 \mathrm{bp}$ of the $C p R a n 1$ gene relative to the start codon. Thus, we concluded that the putative CpRan1-null mutant appeared to be a heterokaryon, consisting of two genetically different nuclei in a common cytoplasm: one for the wild-type and the other for the CpRan1-null mutant allele.
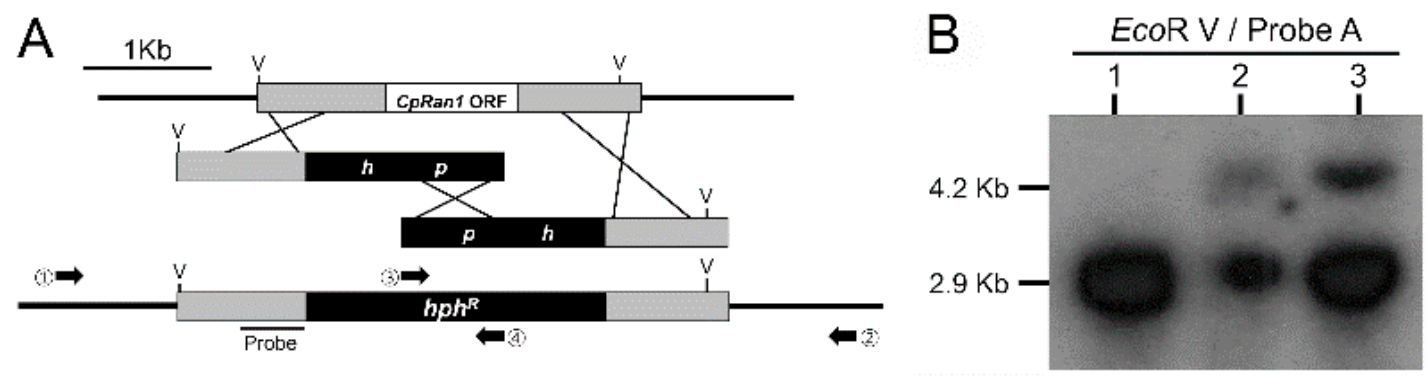

Figure 2. Restriction map and Southern blot analysis of the wild-type EP155/2 and the CpRan1-null mutant. (A) Restriction map of the CpRan1 genomic region and the gene replacement split makers replacing the CpRan1 ORF region with the hygromycin B resistance cassette flanked by 1035-bp and 980-bp fragments as the $5^{\prime}$ - and $3^{\prime}$-flanking regions, respectively. The CpRan1-null mutant with the desired replacement at CpRan1 is represented in the map with the expected changes in the size of the restriction fragments. Flanking regions and expected replacing ORF region are indicated by shadows and open boxes, respectively. $h p h^{\mathrm{R}}$, indicated by the dashed box, represents the hygromycin B resistance cassette. Genes outside the replacement vector are indicated by lines. V represents restriction endonuclease EcoRV. (B) Southern blot analysis of the wild-type EP155/2 strain (lane 1) and two heterokaryotic CpRan1-null mutants (lanes 2, 3; note that in order to compare the intensity of hybridizing bands, two different amounts $(10 \mu \mathrm{g}$ and $20 \mu \mathrm{g})$ of genomic DNA were used for lanes 2 and 3 , respectively.). Enzyme/probe combination is indicated above the line, and the probe A is indicated in the restriction map in the upper panel (A).

\subsection{Heterokaryon Analysis of the Putative CpRan1-Null Mutant}

Since an asexual spore (conidium) of C. parasitica is an aseptate monokaryon, i.e., a single cell with a single nucleus [32], single-spore isolation is the common biological method we used to obtain progeny that have resolved the heterokaryotic state of the putative CpRan1-null mutant. Conidia were harvested from putative CpRan1-null mutant colonies, microscopically inspected (Figure 3), diluted, and 100 conidia were plated on selective PDAmb medium containing $50 \mu \mathrm{g} / \mathrm{mL}$ hygromycin B. No mycelial growth of conidia was observed on selective PDAmb media even when the incubation period was 
extended. When the same spore suspension was plated on the non-selective PDAmb media without hygromycin B, 60 to 80 hygromycin sensitive colonies per plate were observed. We performed a genotype analysis using PCR with gene specific primers of the resulting hygromycin sensitive colonies to confirm the genotype of the single-spore progenies from the germinated conidia. PCR analysis of the single-spore progenies revealed the PCR amplicon that corresponds to the wild-type CpRan1 allele, but not to the CpRan1-null mutant allele (Supplementary Figure S4). These results suggest that the conidia suspension consisted of a mixture of two types of conidia: one viable but sensitive to hygromycin $\mathrm{B}$ and containing the wild-type CpRan1 allele, and the other non-viable and most likely containing the $C p R a n 1$-null mutant allele. These results clearly suggested that the conidia suspension consisted of a mixture of two types of conidia: one was viable but hygromycin B-sensitive containing the wild-type CpRan1 allele and another that was non-viable most likely containing the CpRan1-null mutant allele.
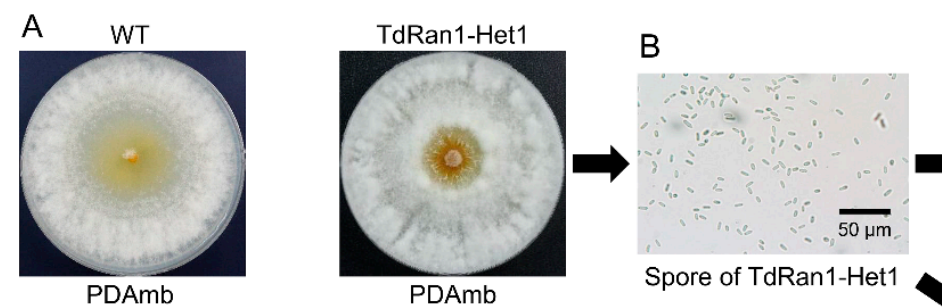

C

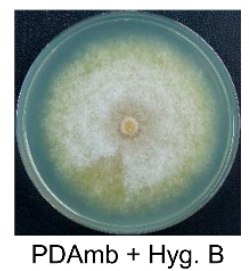

Spore of TdRan1-Het1
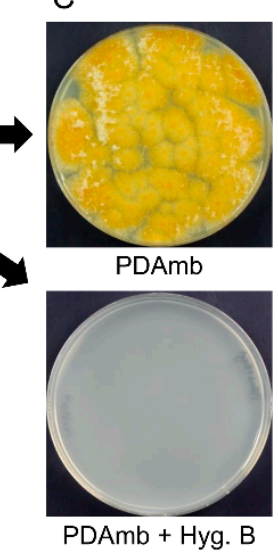

Figure 3. Identification of the CpRan1-null mutant and segregation of conidia of the CpRan1-null mutant strain. (A) The colony morphology after 10 days of culturing is shown. A further extension of the incubation period did not lead to sporulation on PDAmb with hygromycin B. No mycelial growth of conidia was observed on selective PDAmb media even when the incubation period was extended. (B) Conidia harvested from the putative CpRan1-null mutant strain grown on PDAmb for more than 14 days are shown. (C) 100 conidia were spread on PDAmb plates with or without hygromycin B. Strain identifications are provided above the picture. WT and TdRan1-Het1 denote the EP155/2 and heterokaryotic CpRan1-null mutant strains, respectively.

Complementation of the putative $C p R a n 1$-null mutant strain was conducted using protoplasts from the parental heterokaryotic strain and the complementing vector consisting of the wild-type allele of $C p R a n 1$ and the geneticin-resistance selection marker. Complemented strains showing stable geneticin resistance were selected. Conidia were harvested from the selected geneticin-resistant complemented strains and plated on the non-selective media as well as selective media containing hygromycin B and/or geneticin. Colonies displaying both hygromycin B and geneticin resistance were selected, and PCR analysis of subsequent progeny revealed the presence of both the wild-type and CpRan1-null mutant alleles. Colonies showing resistance to geneticin alone or susceptibility to both hygromycin $\mathrm{B}$ and geneticin revealed the presence of only the wild-type CpRan1 allele.

These results suggested that an absence of the essential CpRan1 gene is lethal. We confirmed that the parental mutant strain was a heterokaryon consisting of two different types of nuclei, i.e., one for the wild-type and the other for the CpRan1-null mutant allele.

\subsection{Characteristics of Heterokaryons}

During comparison of cultural characteristics of the parental heterokaryon (TdRan1Het1) with the wild-type EP155/2, there were no differences observed between TdRan-Het1 and the wild type on a non-selective medium (Figure 3A). 
In order to analyze the terminal phenotype of the mutant allele, we microscopically observed the conidial germination (Figure 4). Although a large proportion of conidia germinated and resulted in actively growing young hyphae, there were a large number of residual conidia with the mutant allele showing no signs of germination such as swelling or germ tube formation. These results indicated that $C p R a n 1$ is required from the beginning of fungal cell metabolism and suggested that the nucleocytoplasmic transport by Ran protein is fundamental to affect numerous cellular process.
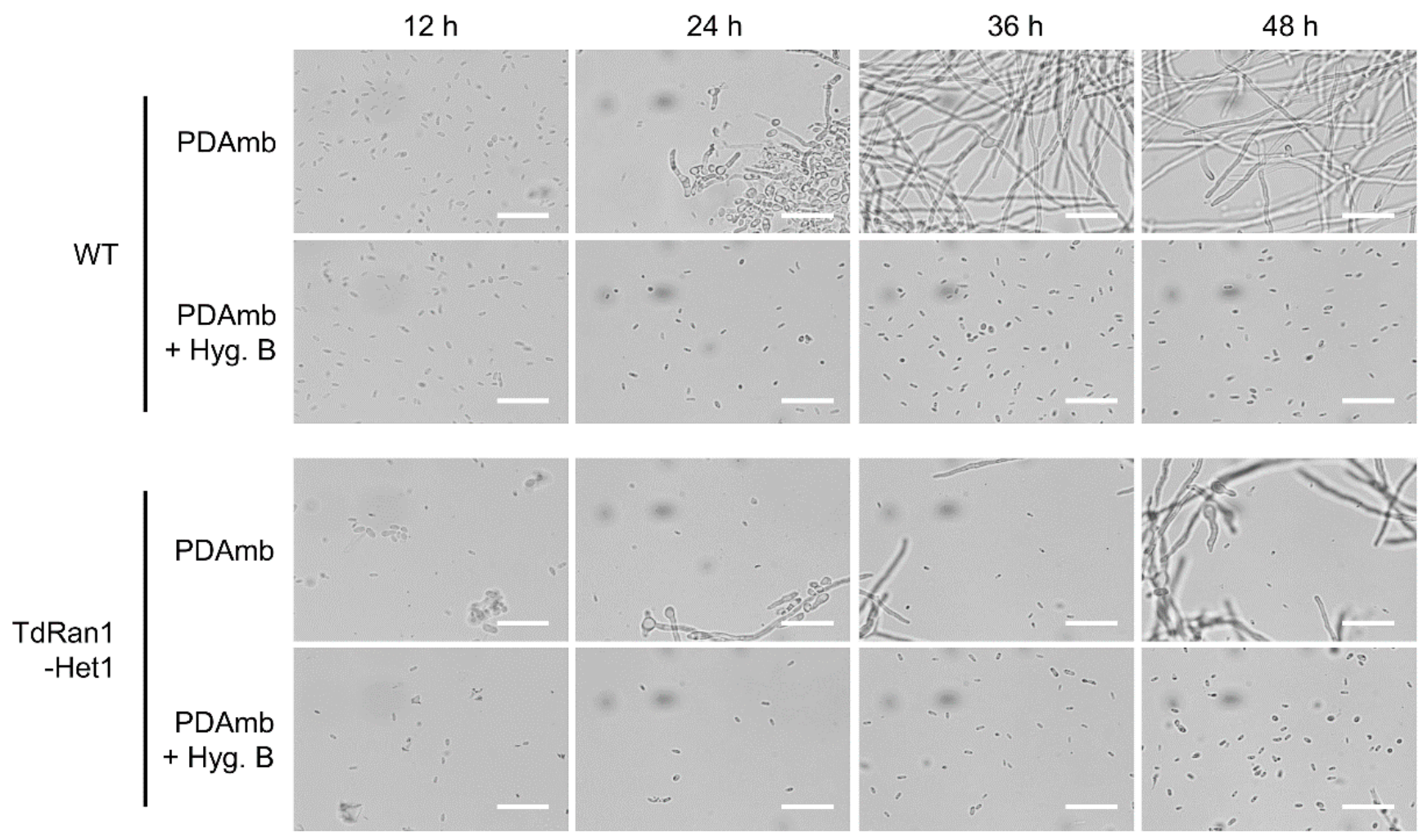

Figure 4. Microscopic observation of germinating conidia. Conidia are observed at every $12 \mathrm{~h}$ until $48 \mathrm{~h}$, and the incubation time is shown above the panel. The strains compared are wild-type EP155/2 (WT) and heterokaryotic CpRan1-null mutant (TdRan1-Het1). The culture media indicated on the left of the panel are potato dextrose broth (PDB) and hygromycin B-supplemented PDB (PDB + Hyg. B). Scale bar $=50 \mu \mathrm{m}$.

Once we have confirmed the existence of the CpRan1-null mutant nuclei, we have tested for any discernable changes in the nuclei of heterokaryon using DAPI staining. As shown in Figure 5, the wild type demonstrated the expected well-spaced single nuclei within a single cell demarcated by septa, while the heterokaryon tended to have more than one nucleus per cell. However, we observed no difference in the intensity and foci between the DAPI-stained nuclei in the heterokaryon and the wild type, suggesting that the protein product of the CpRan1 gene from the wild-type nuclei was able to in trans complement the absence of the Ran protein of mutant nuclei. These results suggest that the $C p R a n 1$ gene is critical from the earliest stages of hyphal growth and that the absence of the CpRan1 gene, and the consequential absence of a nucleocytoplasmic transport system, affects all of the Ran protein's functions, including nuclear transportation, nuclear assembly, mRNA processing, and cell cycle control [21-25]. 


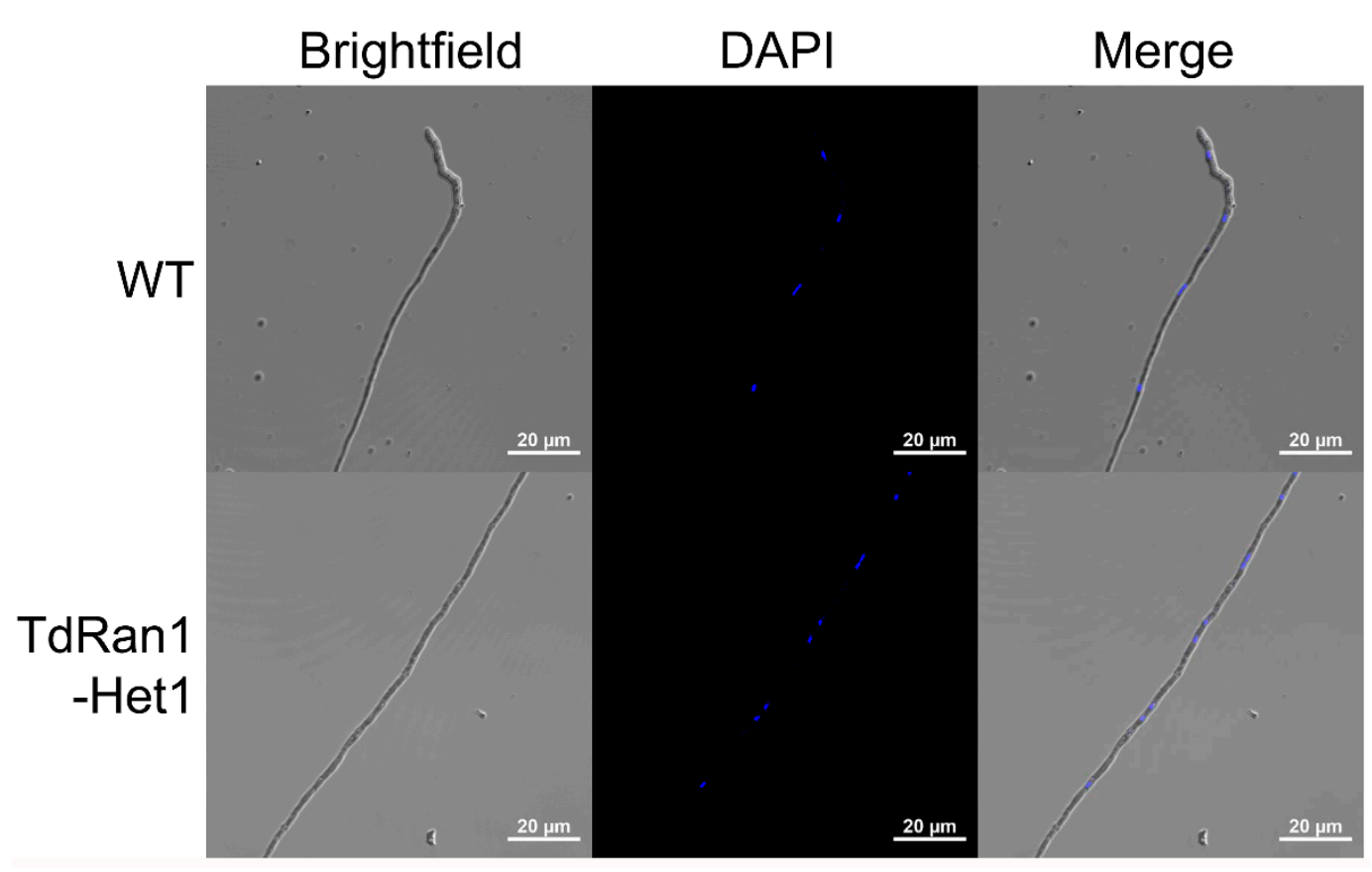

Figure 5. DAPI-stained nuclei of wild-type and the CpRan1-null mutant mycelia. Brightfield: Light microscopic images of mycelia. DAPI: DAPI-stained images of corresponding brightfield mycelia. Merge: Merged images are shown. Strains are indicated on the left. WT and TdRan1-Het1 indicate wild-type EP155/2 and heterokaryotic CpRan1-null mutant strain, respectively.

Since DAPI staining suggests the maintenance of the integrity of the nuclei of both wild-type as well as mutant strains and the genotype frequency in a heterokaryon can vary depending culture conditions, we examined the forced changes in the genotype frequency of heterokaryon progeny. As shown in Figure 6, we cultured the heterokaryon strain on selective medium and passaged it every fifth day, forcing the heterokaryon to maintain the high ratio of the CpRan1-null mutant nuclei. Initially, 30\% of plated conidia did not germinate, i.e., were lethal, and a ratio of no less than $40 \%$ remained thereafter. When the heterokaryon was successively cultured on non-selective medium, the ratio of CFU gradually increased to $90 \%$ and remained. Thus, depending on the culture conditions, the ratio of viable to nonviable conidia most likely representing wild-type nuclei to CpRan1null mutant nuclei could be varied, ranging from 60 to $90 \%$. However, depending on the genotype frequency, no significant changes in the colony morphology of the heterokaryon were observed.

Hypovirus CHV1 transfection via hyphal fusion was performed by co-culturing the CHV1-infected hypovirulent UEP1 strain and the heterokaryon. Changes in the colony morphology of the CHV1-recipient heterokaryon were observed during the co-culture. Mycelia showing a different morphology were transferred on a selective medium. After the successive transfer of hyphal tips on a selective medium, the presence of CHV1 in the resulting heterokaryon was confirmed by dsRNA preparation followed by agarose gel electrophoresis. Compared to the CHV1-infected UEP1 strain, the CHV1-infected heterokaryon displayed similar viral symptoms such as reduced pigmentation and conidiation (Figure 7). No changes in CHV1 titer were observed in the CHV1-infected heterokaryon. 

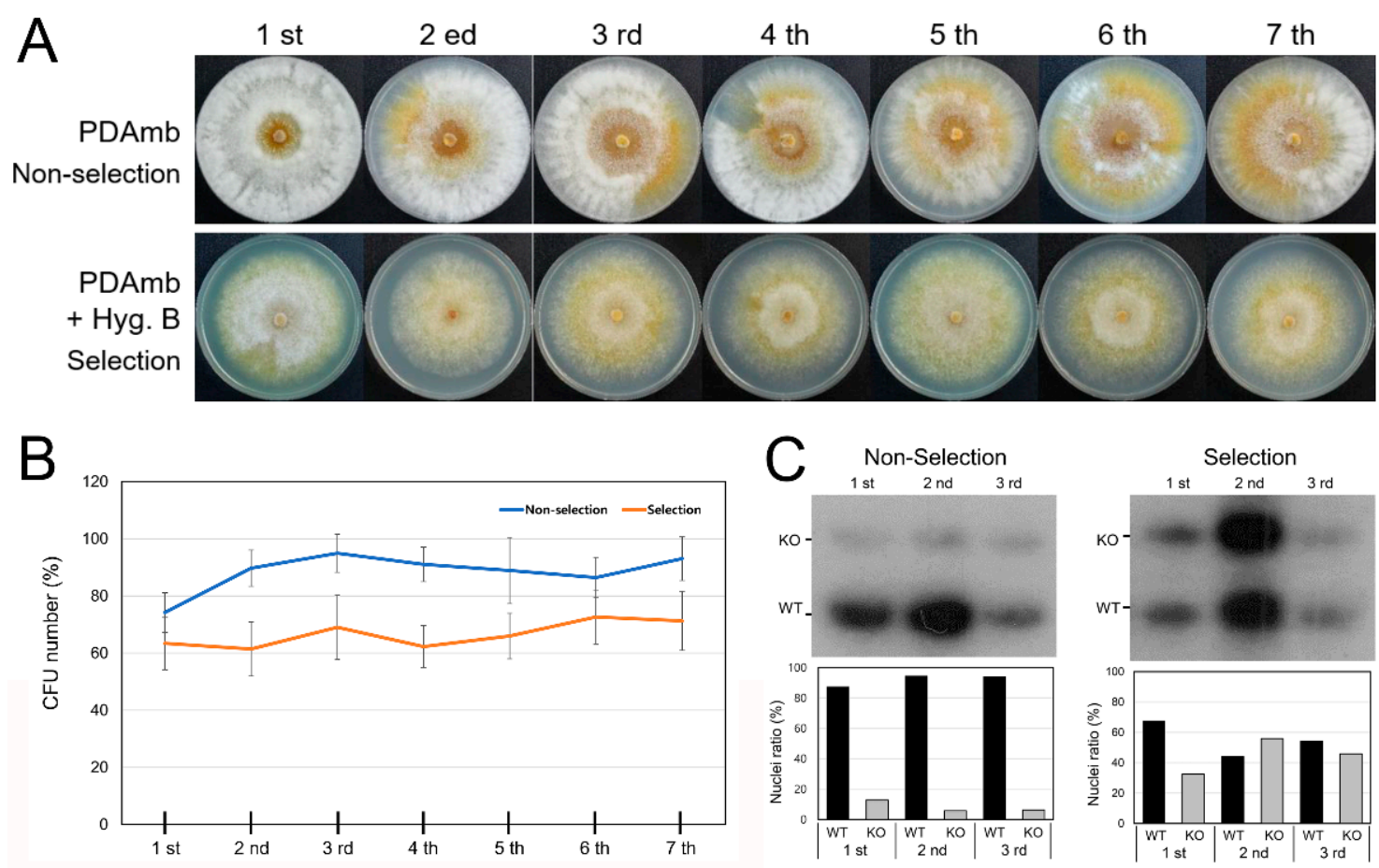

Figure 6. Colony morphology of heterokaryon with and without continuous hygromycin B selection pressure with continuous passage. (A) Morphology of CpRan1-null mutant (TdRan1-Het1) on nonselective, PDAmb, (upper panel) and selective, PDAmb + Hyg. B; PDAmb supplemented with hygromycin B, (lower panel) media. The number of passages was displayed on the top of the plate. (B) Number of germinating spores (CFU) harvested from TdRan1-Het1. (C) Southern blot analysis of wild-type (WT) and CpRan1-null (KO) nuclei after the successive cultures on non-selective and selective media (upper panels). Densitometry of the hybridizing bands in the corresponding upper panel using Image J. PDAmb and PDAmb + Hyg. B are used for the non-selective and selective media, respectively.

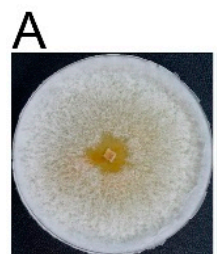

EP155/2

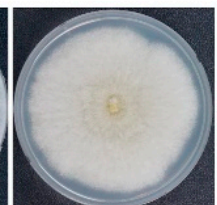

UEP1
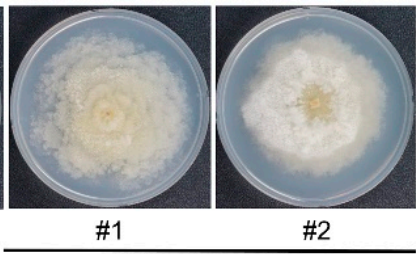

CHV1-TdRan1-Het1

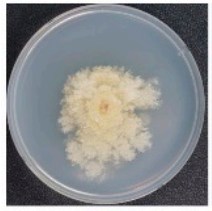

\#3

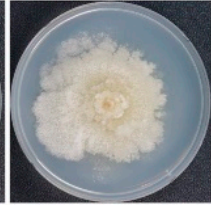

\#4

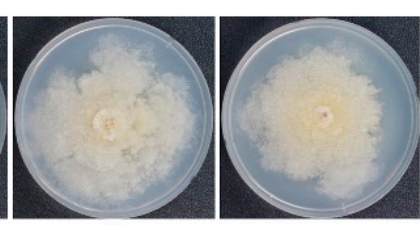

CHV1-TdRan1-Het1

Figure 7. Effect of CHV1 infection to heterokaryons. (A) Colony morphology of CHV1-infected heterokaryotic C $p$ Ran1null mutant. Strains included are virus-free (EP155/2), a virus-infected isogenic strain (UEP1), and six representative CHV1-infected heterokaryotic progenies (CHV1-TdRan1-Het1 \#1-\#6). Strains are marked at the bottom of the plate. (B) Gel electrophoresis of dsRNA isolated from $0.1 \mathrm{~g}$ of lyophilized mycelia.

\subsection{Functional Analysis of the Essential CpRan1 Gene Using Heterokaryons}

Heterokaryosis is an efficient biological feature to rescue lethal or recessive genotypic nuclei with the help of wild-type nuclei. In this way, various chimeric structures of the corresponding essential gene are transferred to the mutant nuclei. With nuclei segrega- 
tion using single-spore isolation, it is possible to analyze whether the specific domains or residues of the protein product of an essential gene are necessary for the associated biological function. In this study, we made use of the CpRan1 heterokaryon (TdRan1-Het1) to analyze the biological function of important amino acid residues. We conducted in silico analysis to select amino acid residues, which are conserved and suggested as being involved in representative post-translational modifications. Four amino acid residues thought to be involved in succinylation (K21), ubiquitination (K97), ubiquitination (K121), and phosphorylation (S151), were selected and analyzed for their importance to the protein product of the CpRan1 gene. Various complementing vectors with a point mutation of a target amino acid to alanine were used to transform TdRan1-Het1. The resulting complemented transformants were analyzed for the presence of transformed conidia with resistance to both selection markers of hygromycin B as well as geneticin (Figure 8). For each construct, at least 60 geneticin-resistant transformants were selected and cultured on PDAmb and PDAmb supplemented with hygromycin B or geneticin (Table 1). Then, transformants showing resistance to both hygromycin $B$ and geneticin were further cultured for harvesting spores, and the resulting spores were plated on PDAmb and PDAmb supplemented with hygromycin B or geneticin (Supplementary Figure S5A). Except for complementation with genomic DNA, PCR amplification of the various transforming CpRan1 genes, which use primers from introns, was applied to rule out the possible contamination of heterokaryotic mycelial fragments (Supplementary Figure S5B). In addition, PCR amplification of the transforming gene followed by sequencing was conducted to verify the corresponding gene. Among tested specimens, genomic as well as cDNA clones of the CpRan1 gene were able to complement the CpRan1-null mutant gene to result in canonical colony morphology. The resulting complemented strains showed a similar phenotype as the wild-type EP155/2. Among those tested with the chimera of the CpRan1 gene, $\mathrm{K} 97 \mathrm{~A}$ is the only one to complement the CpRan1-null nuclei, i.e., conidia growing on both hygromycin B- and geneticin-supplemented media were obtained. Thus, we concluded that $\mathrm{K} 97$ residue was not necessary for the function of the CpRan1 gene and that the K97A mutation was able to support the fungal growth. However, changes in K21, K121, and S151 residues resulted in a lethal phenotype suggesting that these residues are necessary for the function of the CpRan1 gene. Additionally, transformants complemented by K97A showed the characteristic colony morphology of the wild-type EP155/2, indicating that K97 residue is not essential and does not play any role in the full function of the protein product of the CpRan1 gene. Interestingly, the gene from M. musculus was able to complement the mutant nuclei to grow a colony, but the complemented strain showed a slightly different colony morphology from the wild type in which delayed pigmentation was observed.

Table 1. Numbers of transformants using various complementing CpRan1 vectors on different selection media and numbers of resulting hygromycin B-resistant transformants used for spore harvesting and single-spore analysis.

\begin{tabular}{cccccc}
\hline \multirow{2}{*}{$\begin{array}{c}\text { Vector Constructs in } \\
\text { Figure } 8\end{array}$} & \multicolumn{2}{c}{ Number of Transformant } & \multicolumn{3}{c}{ CFU } \\
\cline { 2 - 6 } & $\begin{array}{c}\text { PDAmb } \\
\text { Geneticin }\end{array}$ & $\begin{array}{c}\text { PDAmb + } \\
\text { Hyg. B }\end{array}$ & PDAmb & $\begin{array}{c}\text { PDAmb + } \\
\text { Geneticin }\end{array}$ & $\begin{array}{c}\text { PDAmb + } \\
\text { Hyg. B }\end{array}$ \\
\hline gDNA & 280 & 154 & 23 & 23 & 17 \\
CDNA & 68 & 8 & 8 & 8 & 6 \\
K21A & 60 & 0 & - & - & - \\
K97A & 74 & 7 & 7 & 7 & 5 \\
K121A & 65 & 0 & - & - & - \\
S151A & 60 & 0 & - & - & - \\
Mouse & 80 & 9 & 9 & 9 & 5 \\
\hline
\end{tabular}




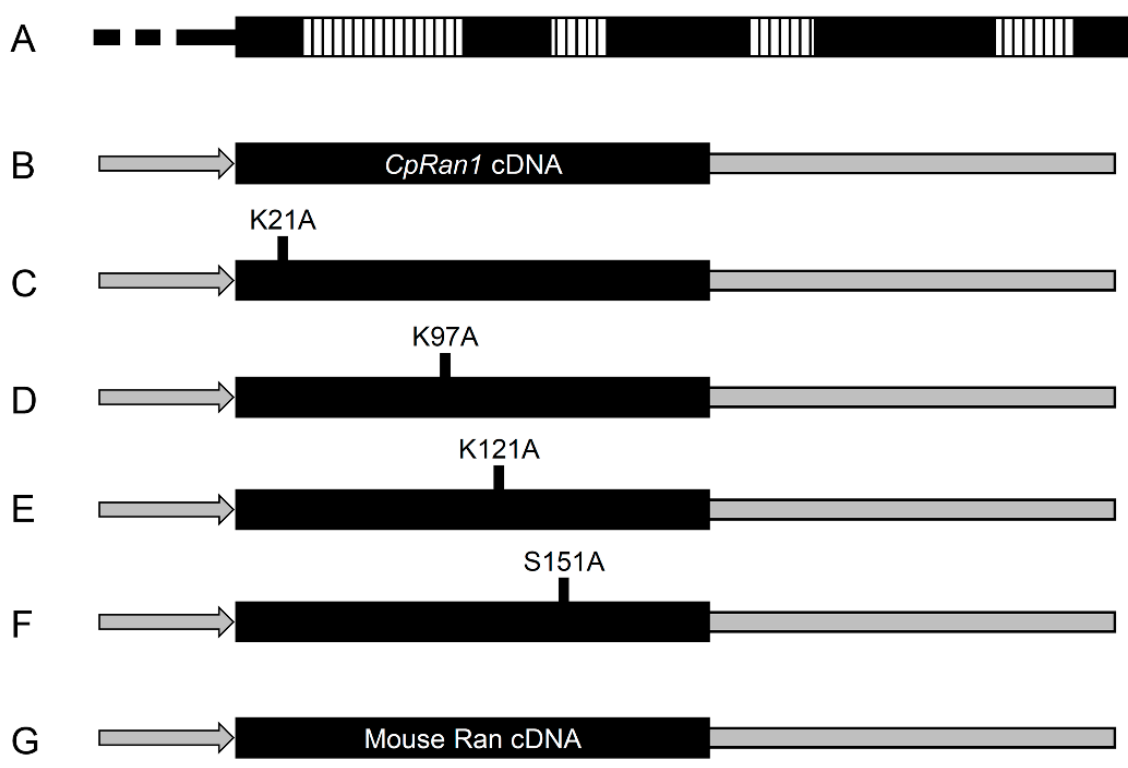

Figure 8. Schematic representation of the complementing vectors. (A) Full-length genomic DNA of the CpRan1 gene was used as a control; vertical dashed lines in the genomic DNA represent introns. (B-F) A series of vector constructs containing various point mutations of $C p R a n 1$ were constructed using cDNA of the $C p R a n 1$ gene and an expression cassette consisting of a strong constitutive cryparin promoter (p188) and trpC terminator. (G) A cDNA clone of Ran gene from mouse (GenBank No. AAH83356.1) was used for the heterologous expression.

\section{Discussion}

Ran is an essential component of the nucleocytoplasmic transport and is implicated in diverse cellular metabolic processes [21-25]. In silico analysis of the genome database of C. parasitica demonstrates that there is only one Ran gene, CpRan1, in the genome of C. parasitica. Based on the discrepancy between proteomic and transcription analysis, it appears that the regulation of the expression of the CpRan1 gene is multilayered and numerous factors can affect $C p R a n 1$ expression.

It is not uncommon for proteomic analysis to show different expression patterns from transcriptomic analysis [20], especially when applied to genes regulated at posttranscriptional, translational, and post-translational levels [41,42]. In our expression analysis results that used cultures under the same conditions, i.e., $24 \mathrm{~h}$ after the transfer to the TA-supplemented media, the qRT-PCR analysis showed a slight but significant increase in the C $p$ Ran 1 transcripts, while the corresponding protein spot displayed a larger two-fold decrease [20]. Moreover, dramatic changes in the accumulation of CpRan1 transcripts were observed by the CHV1 infection under the same culture conditions. Northern blot analysis at $24 \mathrm{~h}$ after the transfer to the TA-supplemented media was consistent with the qRT-PCR analysis, suggesting that the increase in the amount of $C p R a n 1$ transcripts was not mere measurement noise. The differences between mRNA and protein expression results suggested a regulatory process after the transcriptional level. Such regulatory processes are central to explain the abundance of the protein product of the CpRan1 gene, which is critically modulated by the CHV1 infection and TA supplementation. Additionally, upregulation of the CpRan1 transcripts by the CHV1 infection were greater on solid medium than in liquid culture conditions, confirming that $C p R a n 1$ gene expression is also seriously affected by different culture conditions. In sum, our results indicate that the regulation of CpRan1 gene expression is complex and implicates numerous factors at various levels.

The high ratio of the wild-type nuclei over the CpRan1-null mutant nuclei was interesting. The high ratio of the wild-type nuclei of the CpRan1 heterokaryon (TdRan1-Het1) was maintained on the non-selective media $(90 \%)$ and even after the successive cultures on selective media ( $>60 \%$ ). This high ratio wild-type C $p$ Ran 1 nuclei was $40 \%$ to $70 \%$ greater than those of other essential genes such as $C p C d c 48$ and $C p R b p 1[28,31]$. This is indicative of how C. parasitica requires a large amount of protein product from the CpRan1 gene to 
fulfill its fundamental metabolic purpose, which includes nucleocytoplasmic transport. This may simply imply how much gene product is required to maintain the heterokaryon, which indicates that many genes are essential, but the amount of each gene product varies to maintain normal cell metabolism.

A heterokaryon is defined by the presence of two or more genetically different nuclei in a common cytoplasm. It is a unique genetic characteristic of fungi, which allows it to maintain and proliferate the nuclei of recessive genotype with the help of proficient wild-type nuclei. Utilizing the CpRan1 heterokaryon, we were able to proliferate CpRan1null mutant nuclei and complement in trans the CpRan1-null mutant nuclei with various chimeric constructs of the CpRan1 gene. Our complementation analysis using the heterokaryon and various chimeric gene constructs is a proof-of-concept experiment that validates the feasibility of functional analysis of amino acid residues within the protein product of an essential gene. This differs from our previous study in which we illustrated that heterokaryons can be used to determine the necessity of specific protein domains through a domain swapping approach and did not examine the role of specific amino acid residues [28]. Among the four post-translationally modified amino acid residues suggested in silico, our complementation verified that three residues of K21, K121, and S151 played an important role in the biological functioning of the Ran protein. While further studies on the nature of modification will be required to confirm and refine this method, our results suggest that the post-translational modification of $C p R a n 1$, as predicted in silico, does exist and is important to biological functioning. It is possible that the failure of complementation using various chimeric constructs is the consequence of using a heterologous strong constitutive, instead of a native, promoter to express the CpRan1 gene. However, the same strong constitutive promoter was able to complement the CpRan1-null mutant to drive full-length cDNA expression. The strong constitutive promoter was also used for the successful expression of the heterologous Ran gene from M. musculus. Ultimately, therefore, we do not attribute the failure to complement with the mutated amino acid residues to the changes in the expression level of the mutated $C p R a n 1$ gene by the strong constitutive promoter, but rather to true changes in the functionality of the altered amino acid residues. In contrast, the K97A mutation did not affect the ability of Ran1 to complement the knock out, suggesting either that the ubiquitination of $\mathrm{K} 97$ predicted in silico did not occur or, if it did, that the post-translational ubiquitination was no longer necessary to biological functioning. In addition, the success of our complementation, which used a heterologous gene such as the Ran gene from M. musculus, is very promising. The Ran protein is evolutionarily conserved to the point that the amino acid sequence of the Ran protein from M. musculus (GenBank No. AAH83356.1) is similar to other mammalian homologues and is identical to the human Ran gene (GenBank No. AAH16654.1). Our heterokaryon therefore has utility in the functional analysis of heterologous genes from other organisms in which it does not exist. Our recent study on the Ran-binding protein gene CpRbp1, the modulator of Ran, demonstrated that the $C p R b p 1$ gene is essential, which is consistent with the current studies showing the necessity of the $C p R a n 1$ gene. In C. parasitica, four essential genes including the CpRan1 gene have been verified by heterokaryon analysis indicating a well-balanced maintenance of genetically engineered-lethal nuclei $[28,30,31]$. This might represent the increased tendency of heterokaryon formation in essential genes in C. parasitica, even though the stable heterokaryon has been reported in nature [29]. Considering heterologous complementation, our heterokaryon analysis using C. parasitica can be further extended to examine the essential genes of other organisms. Our analysis aids the straightforward detection of essential genes, convenient resolution by single sporulation, and balanced proliferation of genetically recessive nuclei.

In our previous studies, we mimicked the host environment by supplementing tannic acid and revealed several genes that were regulated by tannic acid and/or CHV1 [20]. Among them, three genes, $C p C d c 48, C p R b p 1$, and $C p R a n 1$, were verified to be essential [28,31]. Further studies will be required to analyze why these essential genes are affected under the conditions of TA supplementation, which is also known as the host defense barrier. It will also 
be important to understand whether in general it will be necessary to have these essential genes modulated for pathogenesis in other fungi.

In this study, we characterized the putative CpRan1-null mutant to be a heterokaryon consisting of two different types of nuclei carrying either the wild-type or the CpRan1-null mutant allele in its common cytoplasm. Heterokaryon analysis indicated that the Ran gene of the chestnut blight fungus, C. parasitica, is essential, and the loss-of-function is lethal. Microscopic observation of conidia containing mutant nuclei suggested that the CpRan1 gene plays important roles in early germination and is necessary for fundamental cellular function. We demonstrated a successful functional analysis of the CpRan1 gene by complementation of heterokaryons using various chimeric constructs of the CpRan 1 gene. Heterokaryon analysis of the essential gene allow us to increase the likelihood of applying our knowledge of this fungus to the analysis of the structure-function relationship of a conserved essential gene.

Supplementary Materials: The following are available online at https:/ /www.mdpi.com/article/10 .3390/jof7050332/s1, Figure S1: Characterization of CpRan1 gene, Figure S2: Northern blot analysis of CpRan1 in response to hypovirus infection and TA supplementation, Figure S3: PCR amplicons of the heterokaryotic CpRan1-null mutant, Figure S4: PCR amplicons of the hygromycin sensitive colonies from the germinated conidia, Figure S5: Complementing analysis of various chimeric structures of CpRan1 gene.

Author Contributions: Y.-H.K., J.C.: designed the experiments and analyzed data; Y.-H.K., J.C.: prepared the figures and analyzed data; D.-H.K.: supervised the experiments and wrote the manuscript. All authors have read and agreed to the published version of the manuscript.

Funding: This work was supported by NRF grants by NRF-2018R1A2A1A05078682.

Acknowledgments: We would like to thank the Institute of Molecular Biology and Genetics at Jeonbuk National University for kindly providing the facilities for this research.

Conflicts of Interest: The authors declare that they have no competing interests.

\section{References}

1. Van Alfen, N.K. Biology and potential for disease control of hypovirulence of Endothia parasitica. Ann. Rev. Phytopathol. 1982, 20, 349-362. [CrossRef]

2. Van Alfen, N.K.; Jaynes, R.A.; Anagnostakis, S.L.; Day, P.R. Chestnut blight: Biological control by transmissible hypovirulence in Endothia parasitica. Science 1975, 189, 890-891. [CrossRef]

3. Anagnostakis, S.L. Biological control of chestnut blight. Science 1982, 215, 466-471. [CrossRef] [PubMed]

4. Nuss, D.L. Biological control of chestnut blight: An example of cirus-mediated attenuation of fungal pathogenesis. Microbiol. Rev. 1992, 56, 561-576. [CrossRef]

5. Havir, E.A.; Anagnostakis, S.L. Oxalate production by virulent but not by hypovirulent strains of Endothia parasitica. Physiol. Plant Pathol. 1983, 23, 369-376. [CrossRef]

6. Elliston, J.E. Characteristics of dsRNA-free and dsRNA-containing strains of Endothia parasitica in relation to hypovirulence. Phytopathology 1985, 74, 151-158. [CrossRef]

7. Rigling, D.; Heiniger, U.; Hohl, H.R. Reduction of Laccse activity in dsRNA-containing hypovirulent strains of Cryphonectria (Endothia) parasitica. Phytopathology 1988, 79, 219-223. [CrossRef]

8. Rigling, D.; Prospero, S. Cryphonectria parasitica, the causal agent of chestnut blight: Invasion history, population biology and disease control. Mol. Plant Pathol. 2018, 19, 7-20. [CrossRef]

9. Suzuki, N.; Cornejo, C.; Aulia, A.; Shahi, S.; Hillman, B.; Rigling, D. In-Tree behavior of diverse viruses harbored in the chestnut blight fungus, Cryphonectria parasitica. J. Virol. 2021, 95. [CrossRef]

10. Kazmierczak, P.; Pfeiffer, P.; Zhang, L.; Van Alfen, N.K. Transcriptional repression of specific host genes by the mycovirus Cryphonectria hypovirus 1. J. Virol. 1996, 70, 1137-1142. [CrossRef] [PubMed]

11. Kang, H.S.; Choi, J.W.; Park, S.M.; Cha, B.J.; Yang, M.S.; Kim, D.H. Ordered differential display from Cryphonectria parasitica. J. Plant Pathol. 2000, 16, 142-146.

12. Allen, T.D.; Nuss, D.L. Specific and common alterations in host gene transcript accumulation following infection of the chestnut blight fungus by mild and severe hypoviruses. J. Virol. 2004, 78, 4145-4155. [CrossRef]

13. Dawe, A.L.; Segers, G.C.; Allen, T.D.; McMains, V.C.; Nuss, D.L. Microarray analysis of Cryphonectria parasitica Galpha- and Gbetagamma-signalling pathways reveals extensive modulation by hypovirus infection. Microbiology 2004, 150, 4033-4043. [CrossRef] 
14. Deng, F.; Allen, T.D.; Hillma, B.I.; Nuss, D.L. Comparative analysis of alterations in host phenotype and transcript accumulation following hypovirus and mycoreovirus infections of the chestnut bright fungus Cryphonectria parasitica. Eukaryot. Cell 2007, 6, 1286-1298. [CrossRef]

15. Dawe, A.L.; Nuss, D.L. Hypovirus molecular biology: From Koch's postulates to host self-recognition genes that restrict virus transmission. Adv. Virus Res. 2013, 86, 109-147. [PubMed]

16. Churchill, A.C.L.; Ciuffetti, L.M.; Hansen, D.R.; Van Etten, H.D.; Van Alfen, N.K. Transformation of the fungal pathogen Cryphonectria parasitica with a variety of heterologous plasmids. Curr. Genet. 1990, 17, 25-31. [CrossRef]

17. Chen, B.; Choi, G.H.; Nuss, D.L. Attenuation of fungal virulence by synthetic infectious hypovirus transcripts. Science 1994, 264, 1762-1764. [CrossRef] [PubMed]

18. Kim, D.H.; Rigling, D.; Zhang, L.; Van Alfen, N.K. A new extracellular laccase of Cryphonectria parasitica is revealed by deletion of lac1. Mol. Plant Microbe Interact. 1995, 8, 259-266. [CrossRef]

19. Segers, G.C.; Wezel, R.V.; Zhang, X.; Hong, Y.; Nuss, D.L. Hypovirus papain-like protease p29 suppresses RNA silencing in the natural fungal host and in a heterologous plant system. Eukaryot. Cell 2006, 5, 896-904. [CrossRef]

20. Kim, J.M.; Park, J.A.; Kim, D.H. Comparative proteomic analysis of chestnut blight fungus, Cryphonectria parasitica, under tannic-acid-inducing and hypovirus-regulating conditions. Can. J. Microbiol. 2012, 58, 863-871. [CrossRef] [PubMed]

21. Demeter, J.; Morphew, M.; Sazer, S. A mutation in the RCC1-related protein pim1 results in nuclear envelope fragmentation in fission yeast. Proc. Natl. Acad. USA 1995, 92, 1436-1440. [CrossRef]

22. Ryan, K.J.; Michael McCaffery, J.; Wente, S.R. The Ran GTPase cycle is required for yeast nuclear pore complex assembly. J. Cell Biol. 2003, 160, 1041-1053. [CrossRef]

23. Hetzer, M.W.; Walther, T.C.; Mattaj, I.W. Pushing the envelope: Structure, function, and dynamics of the nuclear periphery. Annu. Rev. Cell Dev. Biol. 2005, 21, 347-380. [CrossRef]

24. Liu, H.; Söderhäll, K.; Jiravanichpaisal, P. Antiviral immunity in crustaceans. Fish Shellfish Immunol. 2009, 27, 77-88. [CrossRef]

25. Mirallas, O.; Ballega, E.; Samper-Martín, B.; García-Márquez, S.; Carballar, R.; Ricco, N.; Jiménez, J.; Clotet, J. Intertwined control of the cell cycle and nucleocytoplasmic transport by the cyclin-dependent kinase Pho85 and RanGTPase Gsp1 in Saccharomyces cerevisiae. Microbiol. Res. 2018, 206, 169-176. [CrossRef] [PubMed]

26. Görlich, D.; Kutay, U. Transport between the cell nucleus and the cytoplasm. Annu. Rev. Cell. Dev. Biol. 1999, 15, 607-660. [CrossRef]

27. Alberts, B.; Johnson, A.; Lewis, J.; Raff, M.; Roberts, K.; Walter, P. Intracellular Compartments and Protein Sorting. In Molecular Biology of the Cell, 4th ed.; Garland Science: New York, NY, USA, 2002.

28. Ko, Y.H.; Choi, S.Y.; So, K.K.; Kim, J.M.; Chun, J.; Kim, D.H. Functional analysis of an essential Ran-binding protein gene, CpRbp1, from the chestnut blight fungus Cryphonectria parasitica using heterokaryon rescue. Sci. Rep. 2020, 10, 8111. [CrossRef]

29. Anagnostakis, S.L. A stable heterokaryon of Endothia parasitica. Mycologia 1981, 73, 570-576. [CrossRef]

30. Kim, M.J.; Park, S.M.; Kim, Y.H.; Cha, B.J.; Yang, M.S.; Kim, D.H. Deletion of a hypoviral-regulated cppk1 gene in a chestnut blight fungus, Cryphonectria parasitica, results in microcolonies. Fungal Genet. Biol. 2004, 41, 482-492. [CrossRef]

31. Ko, Y.H.; So, K.K.; Kim, J.M.; Kim, D.H. Heterokaryon analysis of a Cdc48-like gene, CpCdc48, from the chestnut blight fungus Cryphonectria parasitica demonstrates it is essential for cell division and growth. Fungal Genet. Biol. 2016, 88, 1-12. [CrossRef]

32. Puhalla, J.E.; Anagnostakis, S.L. Genetics and nutritional requirements of Endothia parasitica. Phytopathology 1971, 61, 169-173. [CrossRef]

33. Rigling, D.; Van Alfen, N.K. Extra- and Intracellular laccases of the chestnut blight fungus, Cryphonectria parasitica. Appl. Environ. Microbiol. 1993, 59, 3634-3639. [CrossRef]

34. Powell, W.A.J.; Van Alfen, N.K. Two nonhomologus viruses of Cryphonectria (Endothia) parasitica reduce accumulation of specific virulence-associated polypeptides. J. Bacteriol. 1987, 169, 5324-5326. [CrossRef]

35. Livak, K.J.; Schmittgen, T.D. Analysis of relative gene expression data using Real-time quantitative PCR and the $2^{-\Delta \Delta C} \mathrm{~T}_{\mathrm{T}} \mathrm{Method}$. Methods 2001, 25, 402-408. [CrossRef] [PubMed]

36. Kim, M.J.; Choi, J.W.; Park, S.M.; Cha, B.J.; Yang, M.S.; Kim, D.H. Characterization of a fungal protein kinase from Cryphonectria parasitica and its transcriptional upregulation by hypovirus. Mol. Microbiol. 2002, 45, 933-941. [CrossRef]

37. Choi, G.H.; Nuss, D.L. Nucleotide sequence of the glyceraldehyde-3-phosphate dehydrogenase gene from Cryphonectria parasitica. Nucleic Acids Res. 1990, 18, 5566. [CrossRef]

38. Goswami, R.S. Targeted gene replacement in fungi using a split-marker approach. Methods Mol. Biol. 2012, 835, $255-269$.

39. Nguyen, Q.B.; Kadotani, N.; Kasahara, S.; Tosa, Y.; Mayama, S.; Nakayashiki, H. Systematic functional analysis of calcium signalling proteins in the genome of the riceblast fungus, Magnaporthe oryzae, using a high-throughput RNA-silencing system. Mol. Microbiol. 2008, 68, 1348-1365. [CrossRef] [PubMed]

40. Osherov, N.; May, G. Conidial germination in Aspergillus nidulans requires RAS signaling and protein synthesis. Genetics 2000, $155,647-656$.

41. Greenbaum, D.; Colangelo, C.; Williams, K.; Gerstein, M. Comparing protein abundance and mRNA expression levels on a genomic scale. Genome Biol. 2003, 4, 117. [CrossRef] [PubMed]

42. Vogel, C.; Marcotte, E.M. Insights into the regulation of protein abundance from proteomic and transcriptomic analyses. Nat. Rev. Genet. 2012, 13, 227-232. [CrossRef] [PubMed] 